\title{
La super-résolution
}

\author{
Guillaume DUPUIS \\ Maître de conférences \\ Centre de Photonique Biomédicale - Centre Laser de l'Université Paris-Sud, \\ Fédération LUMAT FR 2764, 91405 Orsay \\ guillaume.dupuis@u-psud.fr
}

$\mathrm{M}$ algré les avancées considérables permises par les microscopes électroniques et les microscopes à sonde locale, les techniques d'imagerie très largement préférées en sciences de la vie sont à l'heure actuelle toujours des techniques optiques utilisant des associations de lentilles et de miroirs conventionnels et des sources de lumière visible. En effet, les objets d'étude privilégiés en sciences de la vie, les cellules, sont optiquement transparents : seule la microscopie optique fournit une imagerie non-invasive de l'intérieur des cellules en trois dimensions, dans des conditions compatibles avec le vivant. En outre, notamment grâce à des techniques de marquage fluorescent, la microscopie optique permet la détection de constituants cellulaires spécifiques, tels que les protéines, les acides nucléiques ou les lipides. La microscopie optique de fluorescence se révèlerait presque la technique d'imagerie idéale pour étudier les objets vivants si elle était capable de séparer des détails dont la taille est inférieure à $250 \mathrm{~nm}$. Il est malheureusement établi depuis les travaux de I'opticien allemand Ernst Abbe (18401905) que la résolution spatiale de tout instrument d'optique est intrinsèquement limitée par la diffraction de la lumière.

Mathématiquement, cette limite fondamentale est décrite par une fonction appelée la fonction d'étalement du point (PSF pour point spread function) : il s'agit en fait de la réponse du microscope à une source ponctuelle. La PSF permet de déterminer les dimensions typiques du plus petit détail que l'on puisse résoudre grâce à un microscope. Dans le cas d'un objectif de pupille circulaire, la taille transversale $r$ de la PSF est donnée par l'expression : $r=1,22 \frac{\lambda}{2 O N}$, où $O N$ désigne l'ouverture numérique de I'objectif. Axialement, la taille de la PSF est typiquement 3 fois plus grande. Pour un objectif de microscope classique d'ouverture numérique 1,40 et à une longueur d'onde de $500 \mathrm{~nm}$, la PSF dans un milieu biologique a l'allure représentée sur la figure 1.

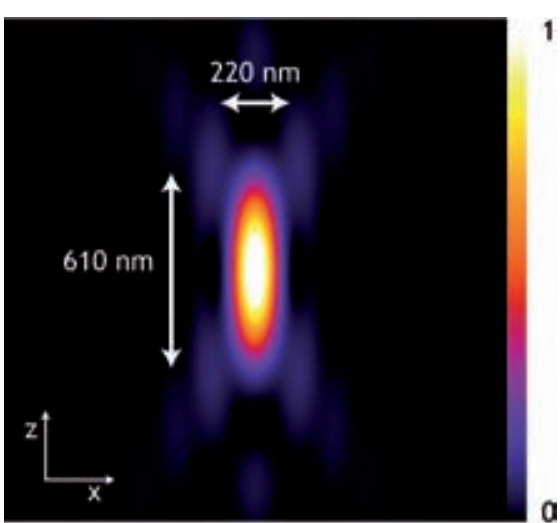

Figure 1. Simulation numérique de la PSF d'un objectif de microscope à immersion $(\mathrm{ON}=1,4)$ pour une longueur d'onde de $500 \mathrm{~nm}$ dans un milieu d'indice 1,5.

La résolution limitée d'un microscope optique est due à la diffraction de la lumière lorsqu'elle se propage au sein de I'instrument, c'est-à-dire sur une distance bien plus grande que la longueur d'onde: on parle d'imagerie en champ lointain. Historiquement, dans les années 1980, les premières tentatives de dépassement de la limite de diffraction ont donc consisté à s'intéresser à l'imagerie en champ proche. Concrètement, il s'agit alors de capter les ondes évanescentes non-propagatives au voisinage de la surface de l'échantillon:on parle alors de microscopie optique en champ proche (SNOM pour scanning nearfield optical microscopy). Dans ce type d'instruments, l'image de l'échantillon est réalisée par balayage mécanique d'une sonde locale constituée d'une fibre optique étirée en pointe, qui apporte la lumière incidente et capte l'onde évanescente. La résolution spatiale obtenue est typiquement de l'ordre de $20 \mathrm{~nm}$. Cela étant, cette technique est intrinsèquement limitée à l'imagerie de surface, et peu compatible avec les échantillons vivants. C'est la raison pour laquelle il s'est avéré indispensable de développer des méthodes d'imagerie de champ lointain qui soient non-limitées par la diffraction.

\section{Comment améliorer la résolution spatiale en microscopie tout en respectant la limite de diffraction?}

En champ lointain, les performances de la microscopie optique de fluorescence peuvent être légèrement améliorées en configuration confocale ou multiphotonique. La microscopie confocaleà balayage laser repose sur l'utilisation d'un faisceau laserfocalisé pourl'excitation et le positionnement d'un trou de filtrage devant le détecteur (cf. figure 2). En principe, il est possible d'améliorer alors la résolution spatiale d'un facteur $\sqrt{ } 2$. En microscopie multiphotonique, l'absorption non-linéaire confine également la taille effective de la PSF d'excitation. Toutefois, ce gain est partiellement compensé par l'augmentation de la longueur d'onde d'excitation (typiquement le proche infrarouge en microscopie biphotonique). Ainsi, à défaut d'une amélioration sensible de la résolution, le principal avantage des approches confocale ou multiphotonique est la réduction drastique de la fluorescence issue des plans hors-focus, qu'on appelle aussi sectionnement optique. 


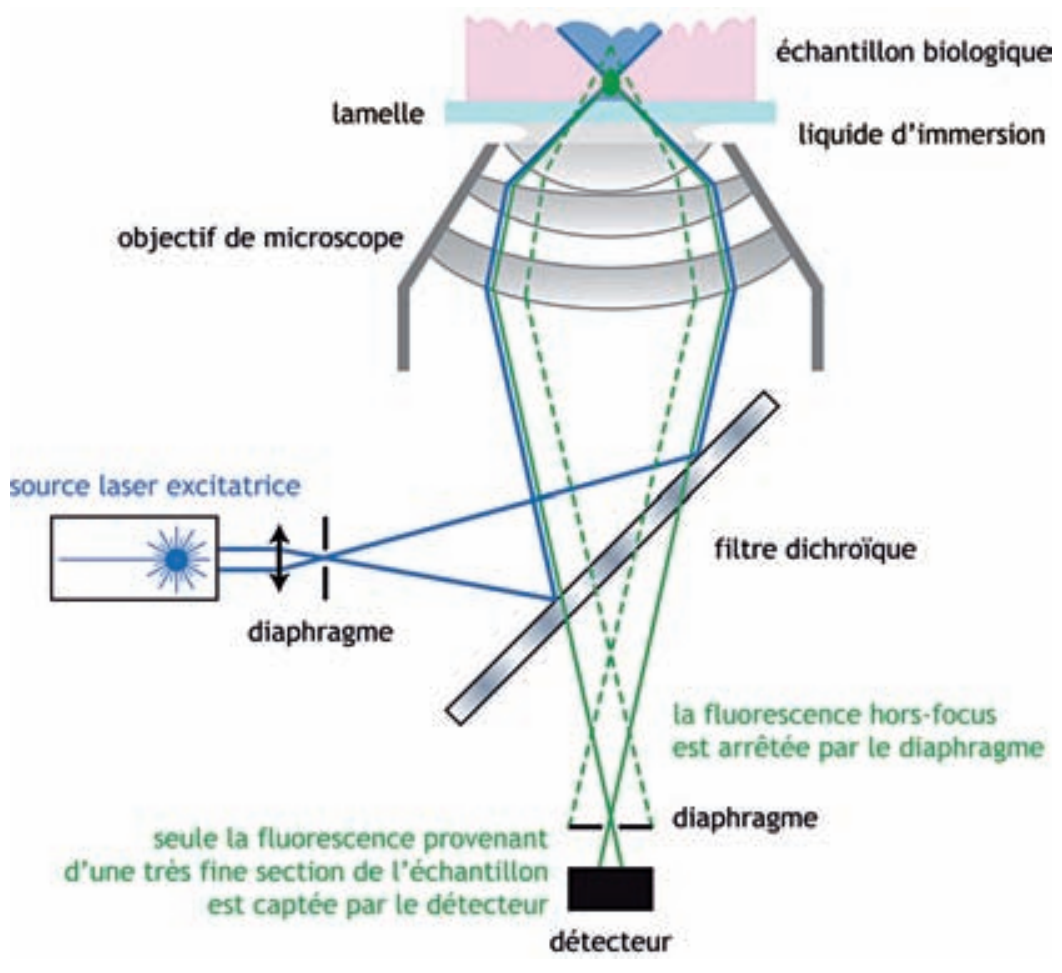

Figure 2. Principe de la microscopie confocale.

Deux types de stratégie en champ lointain améliorent encore davantage la limite de résolution en microscopie optique de fluorescence :

- les techniques utilisant le confinement spatial des ondes évanescentes (microscopie TIRF) ;

- les techniques d'illumination structurée qui consistent à récupérer les hautes fréquences spatiales habituellement coupées par la fonction de transfert du microscope en illumination uniforme.

\section{Microscopie de fluorescence en réflexion totale interne (TIRF)}

L'utilisation des ondes évanescentes pour l'imagerie d'objets biologiques endeçà de la limite de diffraction n'est pas l'apanage des techniques de champ pro che optique. Ainsi, la microscopie de fluorescence en réflexion totale interne TIRF (pour total internal reflection fluorescence microscopy) utilise un mode d'excitation par ondes évanescentes : la fluorescence se trouve confinée à une très fine section optique de l'échantillon, immédiatement adjacente à la lamelle.

Comme le montre la figure 3 , le confinement spatial de l'excitation repose sur le phénomène physique de la réflexion totale interne. Les échantillons biologiques généralement observés ont un indice de réfraction proche de celui de l'eau $(\sim 1,33)$ et ils reposent sur une lamelle de verre d'indice plus élevé $(\sim 1,5)$. À l'interface entre le verre et l'échantillon, sous réserve que la lumière excitatrice provienne du milieu d'indice élevé (le verre), la condition sur les indices est vérifiée pour permettre l'existence du phénomène de réflexion totale interne. Cela étant, une partie du champ incident est transmise à l'échantillon sous forme d'une onde évanescente parallèle à l'interface et dont l'amplitude décroît exponentiellement avec la profondeur. La profondeur de pénétration du champévanescent est typiquement inférieure à $100 \mathrm{~nm}$, soit une valeur nettement inférieure à la limite de diffraction axiale.

La microscopie TIRF permet donc de visualiser de façon très sélective les zones de contact entre la cellule et la lamelle. Les images obtenues présentent de multiples qualités : elles bénéficient tout d'abord d'un très faible bruit de fond, puisque les fluorophores situés dans les couches profondes de l'échantillon ne sont pas excités. De plus, il s'agit d'une technique

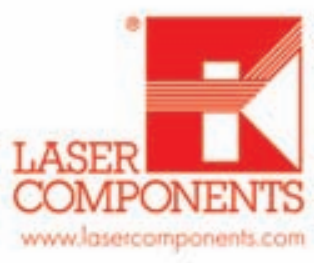

\section{En plein sur la cible}

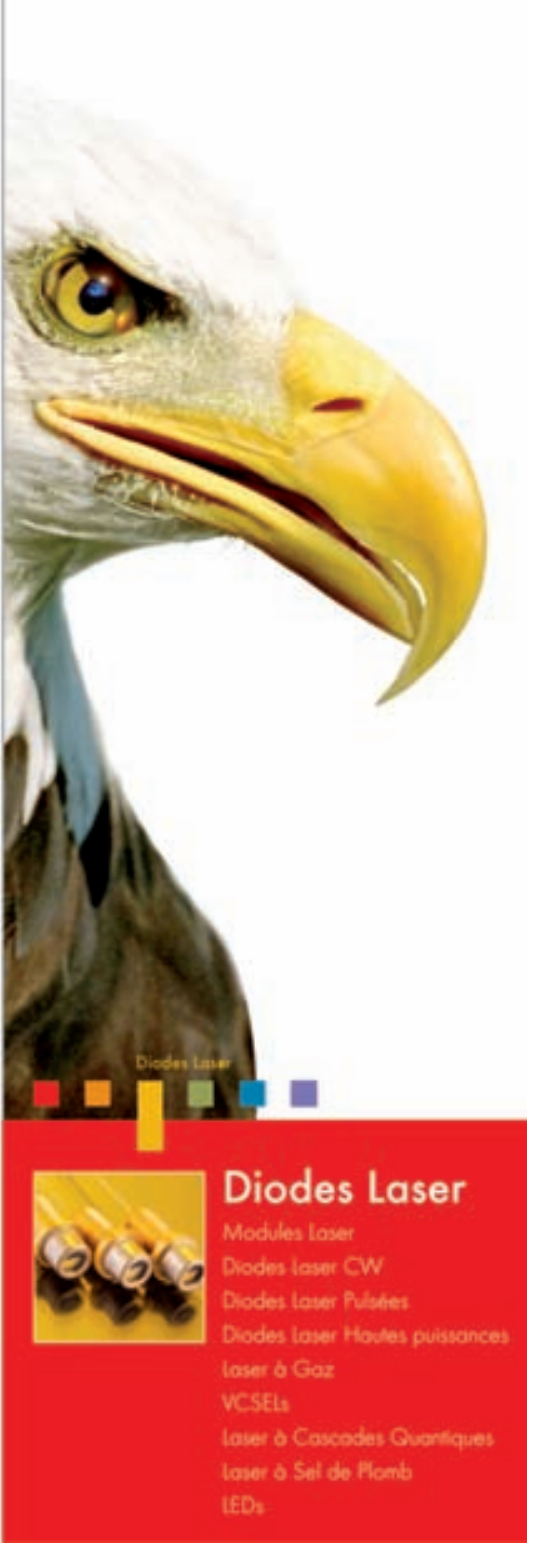

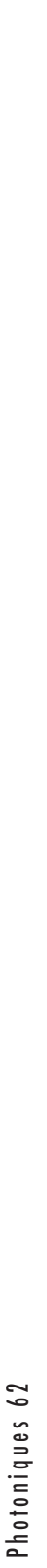


plein-champ, c'est-à-dire sans balayage : les images peuvent être enregistrées à haute cadence. Il est donc possible d'étudier avec une très grande résolution axiale et en temps réel les évènements se produisant à la membrane plasmique de cellules vivantes : adhésion cellulaire, dynamique membranaire (endocytose, exocytose), etc. Cela étant, le principal inconvénient de cette technique est qu'il est intrinsèquement impossible de conserver le sectionnement axial dans les profondeurs de l'échantillon.

\section{Microscopie en illumination structurée (SIM)}

Une autre approche permettant à la fois d'effectuer un sectionnement optique en profondeur et d'améliorer la résolution spatiale des images consiste à illuminer l'échantillon avec un motif structuré. Dans cette configuration, les hautes fréquences spatiales de l'échantillon se mélangent avec la fréquence spatiale du motif de l'illumination, ce qui produit une figure de
Figure 3. Principe de la microscopie de fluorescence en réflexion totale interne.
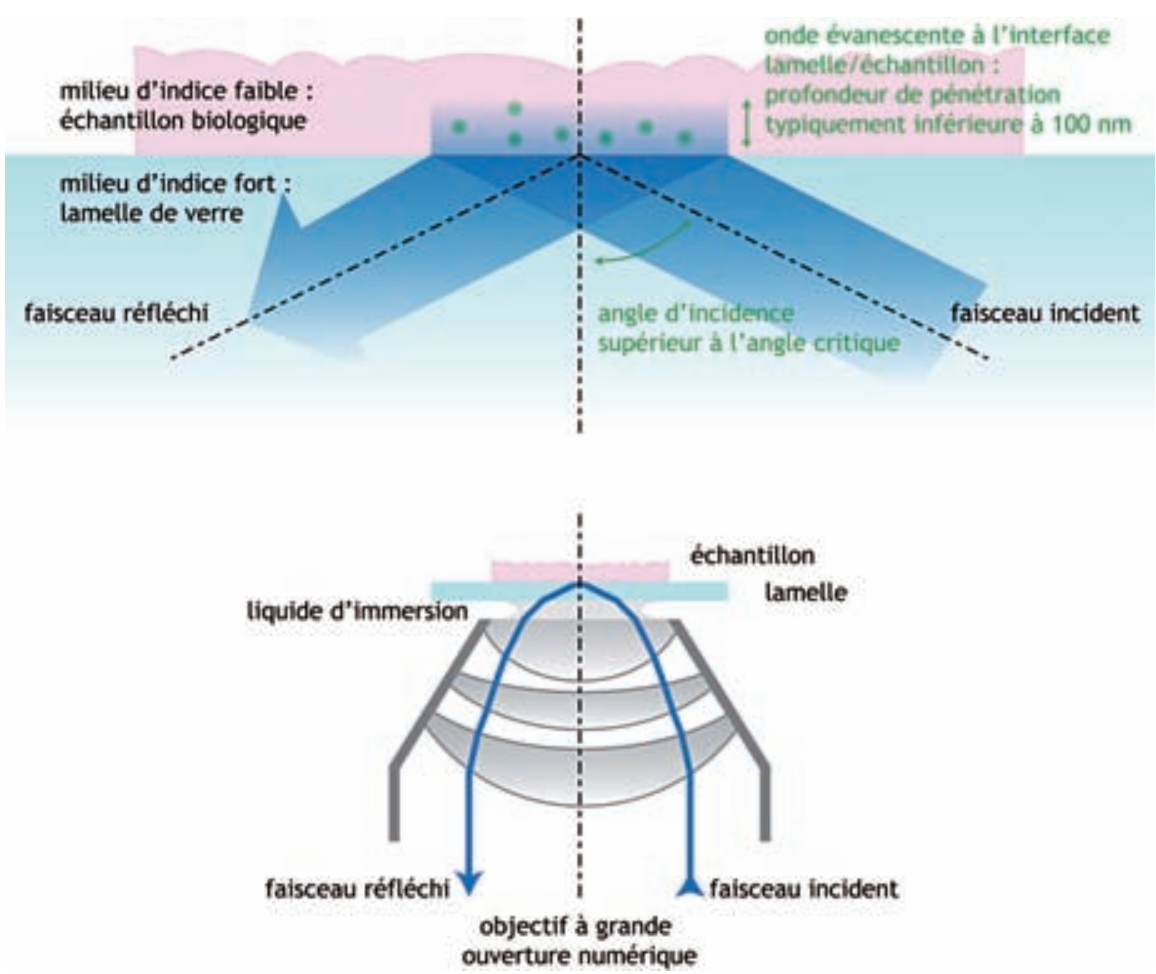

a

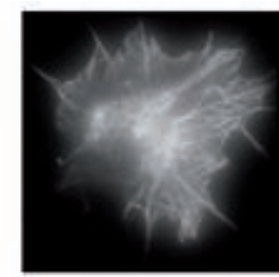

image en épifluorescence (illumination uniforme)

b

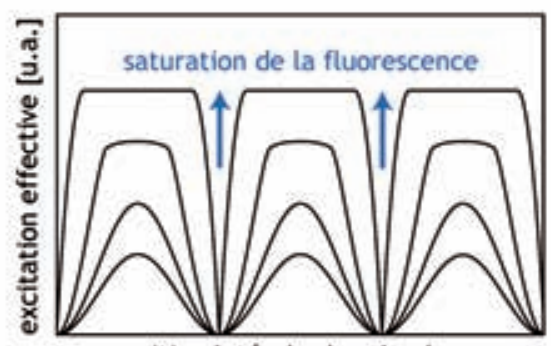

position latérale dans le champ

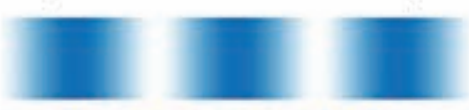

fllumination sinusoïdale (SIM)

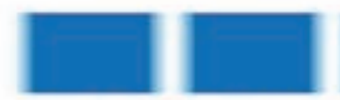

illumination saturée (SSIM)
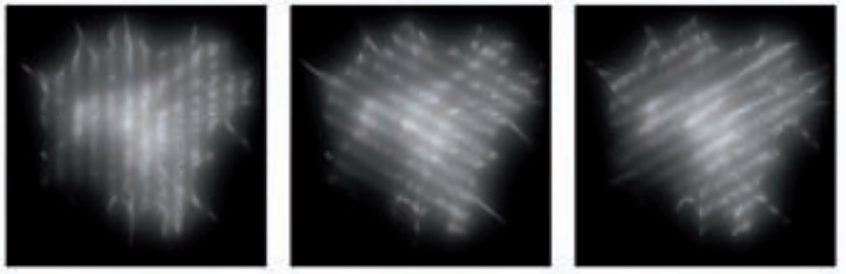

enregistrement de 9 images en illumination structurée ( 3 positions du motif $\times 3$ directions)

$\mathbf{d}$
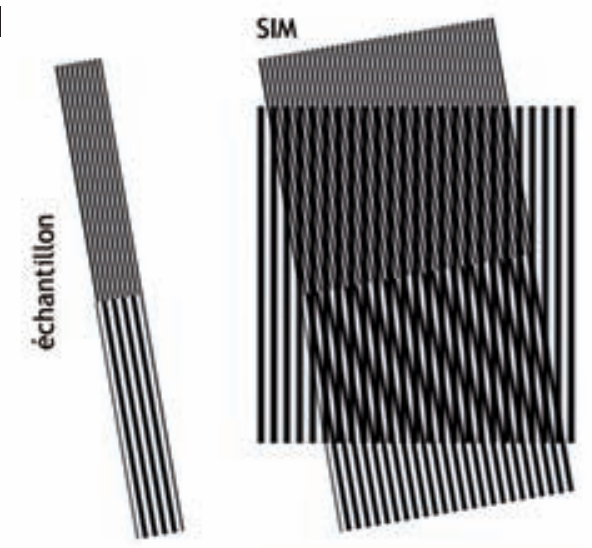

excitation effective

\footnotetext{
Figure 4. Principe de la microscopie en illumination structurée : (a) l'extraction numérique d'une section optique à partir de 9 images structurée repose sur le fait que seule la section optique d'intérêt est modulée à la fréquence du motif d'illumination projeté ; (b) la saturation de la fluorescence entraîne une distorsion du motif d'illumination, initialement sinusoïdal ; ( $)$ dans un échantillon de haute fréquence spatiale, l'effet de Moiré peu visible en illumination sinusoïdale (en haut à gauche) devient visible en illumination saturée (en haut à droite).
} 
Moiré à la fréquence différence plus basse (cf.figure 4).Sicelle-ci n'est pas coupée par la fonction de transfert du microscope, certains détails initialement exclus peuvent être extraits de l'image modulée par traitement numérique. Dans la version de ce concept proposée par Mats Gustafsson en 2000, la structuration de l'illumination est produite en faisant interférer les ordres -1 et +1 d'un réseau de diffraction et l'algorithme de reconstruction nécessite l'enregistrement de 9 images : 3 positions du motif décalées d'un tiers de période, et ce dans 3 directions inclinées de $60^{\circ}$. Cela étant, dans son ensemble, le système reste limité par la diffraction : la fréquence du motif d'illumination et celle de la figure de Moiré doivent rester inférieures à la fréquence de coupure du microscope, de sorte que la résolution spatiale latérale n'est améliorée que d'un facteur 2. La résolution axiale, quant à elle, n'est pas sensiblement différente de celle d'un microscope confocal.

\section{En résumé...}

Le principal avantage commun de ces techniques purement optiques est qu'elles ne reposent pas sur des propriétés photophysiques particulières des fluorophores. Elles peuvent donc être mises en œuvre simplement, avec des sondes fluorescentes classiques. Chacune a cependant ses spécificités : certaines fonctionnent en champ large tandis que d'autres nécessitent un balayage ; certaines améliorent sensiblement la résolution axiale, d'autres interviennent davantage sur la résolution latérale. Généralement, la PSF des instruments de ce type demeure très anisotrope : la résolution latérale reste meilleure que la résolution axiale. C'est la raison pour laquelle, à ce jour, les meilleurs résultats ont été obtenus en combinant l'illumination structurée avec la microscopie $I^{5} \mathrm{M}$ (pour illumination interference and incoherent imaging interference microscopy), qui consiste à illuminer l'échantillon par la figure d'interférence en trois dimensions produite par deux objectifs opposés placés de part et d'autre de celui-ci. Dans cette configuration, la PSF devient quasi-isotrope, ce qui correspond à une résolution spatiale d'environ $100 \mathrm{~nm}$ dans les trois dimensions.

\section{Comment s'affranchir de la limite de diffraction en microscopie?}

Les techniques précédemment évoquées restent néanmoins fondamentalement limitées par la diffraction de la lumière. Afin d'atteindre des résolutions spatiales significativement meilleures, bien au-delà de la limite de diffraction, il faut mettre en œuvre des stratégies qui s'appuient notamment sur les propriétés photo-physiques de certains fluorophores. Schématiquement, on distingue deux approches fondamentalement différentes:

- les techniques utilisant les phénomènes de saturation : ingénierie de la fonction d'instrument (microscopieSTED) et saturation de l'illumination structurée ;

- les techniques reposant sur la localisation successive de fluorophores individuels (microscopies PALM et STORM).

\section{Microscopie STED}

L'idée originale proposée par Stefan Hell en 1994 et démontrée expérimentalement en 1999 consiste à inhiber la fluorescence dans la zone périphérique du volume d'excitation. Cette idée lui permet de dégager un concept général, la microscopie RESOLFT (pour reversible saturable optical fluorescent transition) qu'il met plus particulièrement en application pour mettre au point la microscopie STED (pour stimulated emission depletion), cas particulier pour lequel l'inhibition de la fluorescence est réalisée par émission stimulée.

Dans la figure $5 A$, un diagramme de Jablonski simplifié présente les niveaux d'énergie typiquement impliqués dans les processus photo-physiques en micros copie STED. Le faisceau d'excitation fait passer dans un état d'excitation électronique tous les fluorophores inclus dans le volume correspondant à la tache de diffraction. Le faisceau STED présente une intensité nulle au centre ; il est spatialement superposé avec le faisceau d'excitation et temporellement synchronisé juste avant que l'émission spontanée se produise. Le rôle du faisceau STED est donc d'instantanément dépeupler l'état excité par émission stimulée sur l'ensemble du volume excité, sauf au centre. Étant donné
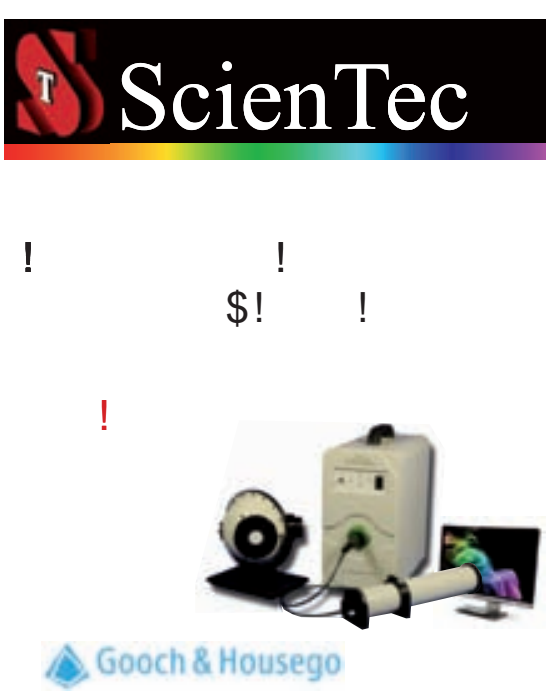

MESURE

, Flux Total

$>$

)

'Température de couleur

IRC

'Pureté

> Couleur
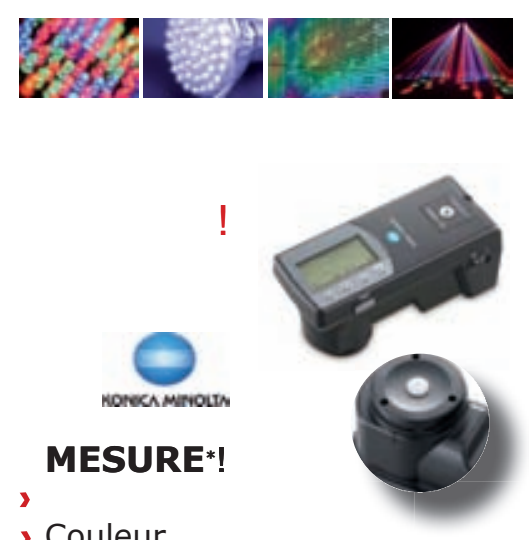

, Couleur

, IRC

, Pureté

, Température de couleur

*Compatible pour sources à LED et autres types de sources lumineuses

\section{Sources de référence}

Luxmètres

Photomètres

Chromamètre

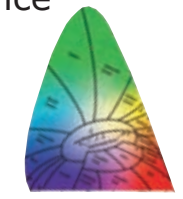

info@scientec.fr / www.scientec.fr 01.64.53.27.00 
a

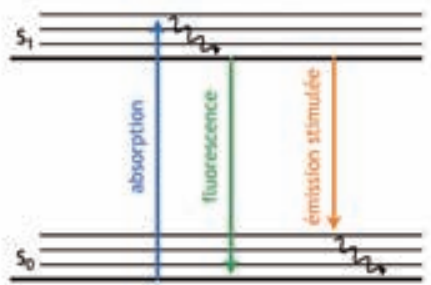

b

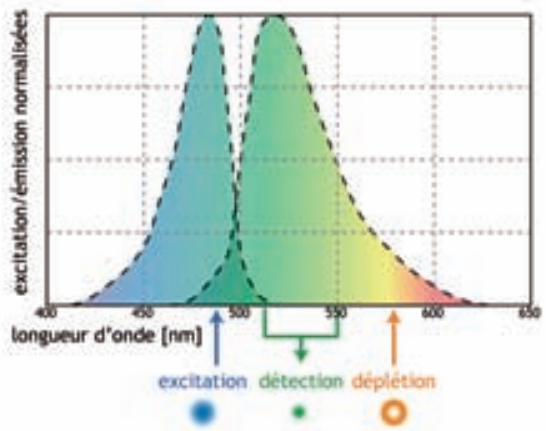

$\mathbf{c}$

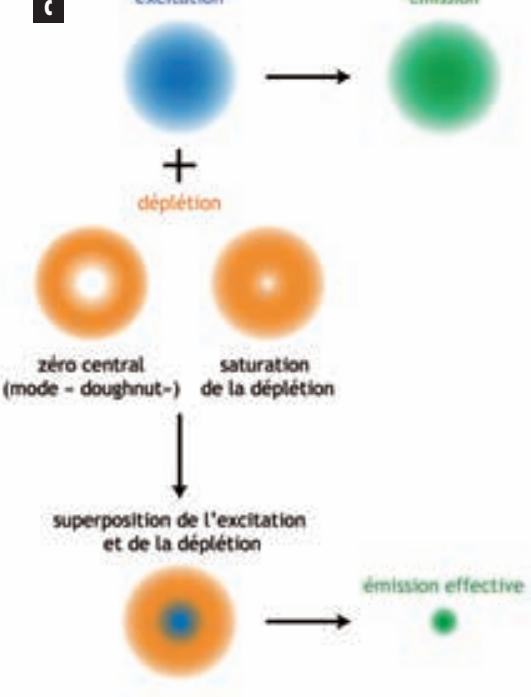

d

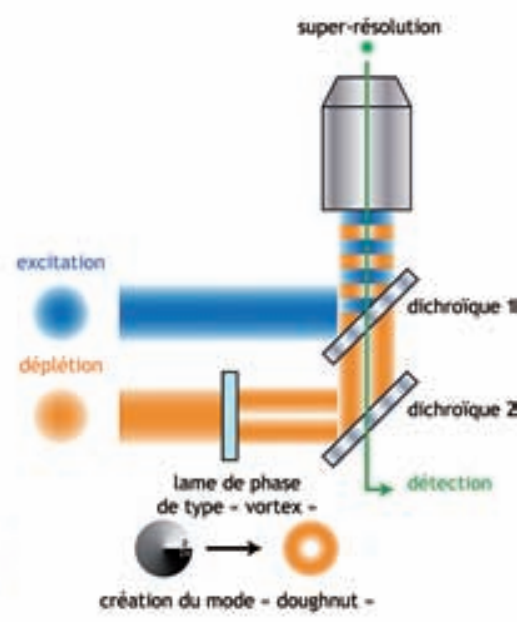

Figure 5. Principe de la microscopie STED : (a) transition énergétique impliquée dans le processus STED ; (b) configuration spectrale du STED pour la protéine fluorescente verte (GFP pour green fluorescent protein); (c) réduction de la PSF d'émission par saturation de la déplétion; (d) schéma de principe d'un microscope STED, la mise en forme du faisceau de déplétion est réalisée par une lame de phase gravée en vortex.

que les photons d'émission stimulée ont une longueur d'onde prédéterminée, ils peuvent être spectralement (voire temporellement) filtrés, apparaissant comme un état sombre sur le détecteur. En re vanche, les molécules présentes au centre de la tache de diffraction sont fluorescentes et leurs positions peuvent être enregistrées avec une résolution non limitée par la diffraction. De cette manière, l'image entière peut ensuite être reconstruite point par point.

La limite de diffraction est dépassée par l'exploitation de la relation non-linéaire entre l'intensité du faisceau STED et le taux de déplétion. En augmentant l'intensité du faisceau STED tout en conservant une intensité strictement nulle au centre, on sature la déplétion de l'état excité : la partie non-déplétée au sein de laquelle la fluorescence se produit se retrouve confinée à une zone de l'espace non-limitée par la diffraction. Théoriquement, la résolution spatiale n'est alors plus limitée que par la puissance disponible dans le faisceau STED, et non par la diffraction. Sur des échantillons pour lesquels les phéno- lules vivantes, il n'est pas possible d'augmenter infiniment la puissance du faisceau de déplétion, ce qui fait que les performances optimales avoisinent plutôt $70 \mathrm{~nm}$.

\section{Microscopie en illumination structurée saturée (SSIM)}

La limite fondamentale de la microscopie en illumination structurée est que la fréquence du motif d'illumination et la fréquence de Moiré résultante doivent respecter la limite de la diffraction : la résolution spatiale n'est améliorée que d'un facteur 2. Mats Gustafsson améliore sa technique en 2005 en saturant la fluorescence. Le motif d'illumination effectif n'est alors plus sinusoïdal (cf. figure $4 B$ ), ce qui a pour effet d'introduire des har moniques élevées dans la structuration. Théoriquement, comme en microscopie STED, la résolution spatiale devient illimitée. Concrètement, les harmoniques élevées sont rapidement noyées dans le bruit et la saturation de la fluorescence favorise les photo-dommages. De plus, dans ces conditions, l'algorithme de traitement nécessite l'enregistrement d'un grand nombre d'images afin d'atteindre la résolution voulue: 81 images sont nécessaires pour atteindre une résolution spatiale latérale de $50 \mathrm{~nm}$ dans les deux directions.

\section{Techniques de super-localisation : microscopies PALM et STORM}

Il est également possible d'améliorer drastiquement la résolution spatiale en microscopie optique de fluorescence en utilisant la localisation successive de molécules uniques, individuellement photoactivées, avec une précision nanométrique. La microscopie PALM (pour photo-activation localization microscopy) proposée par l'équipe d'Eric Betzig et Harald Hess en 2006 et la microscopie STORM (pour Stochastic Optical Reconstruction Microscopy) proposée par l'équipe de Michael Rust en 2006 sont deux exemples de ces techniques de super-localisation, identiques dans le principe, mais n'utilisant pas le même type de fluorophore.

Ces techniques reposent sur l'utilisation de marqueurs fluorescents photo-convertibles dont l'expérimentateur contrôle l'activation stochastique, la lecture, puis la désactivation (cf. figure 6). Au début de l'expérience, les sondes photo-activables présentes dans l'échantillon sont dans l'état «OFF »: elles ne produisent aucun signal de fluorescence. La première étape consiste alors à faire passer une très petite fraction (environ $1 \%$ ) des sondes dans l'état « ON » (photo-activation) grâce à un pulse de lumière de courte longueur d'onde (typiquement $405 \mathrm{~nm}$ ). On enregistre alors une 
structure intracellulaire subdiffraction à imager
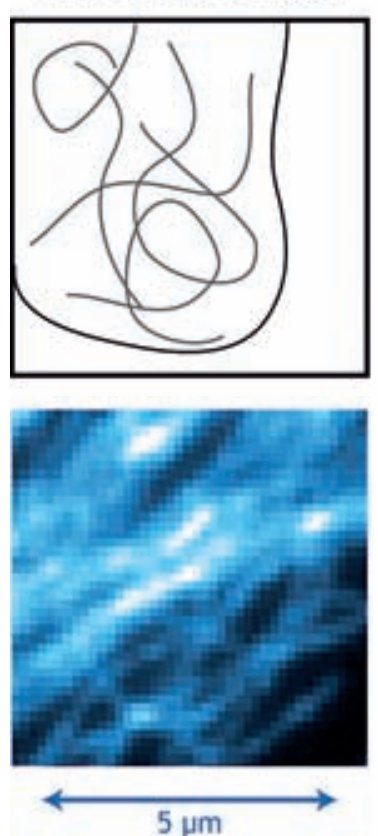

localisation sucessive des fluorophores individuellement activés
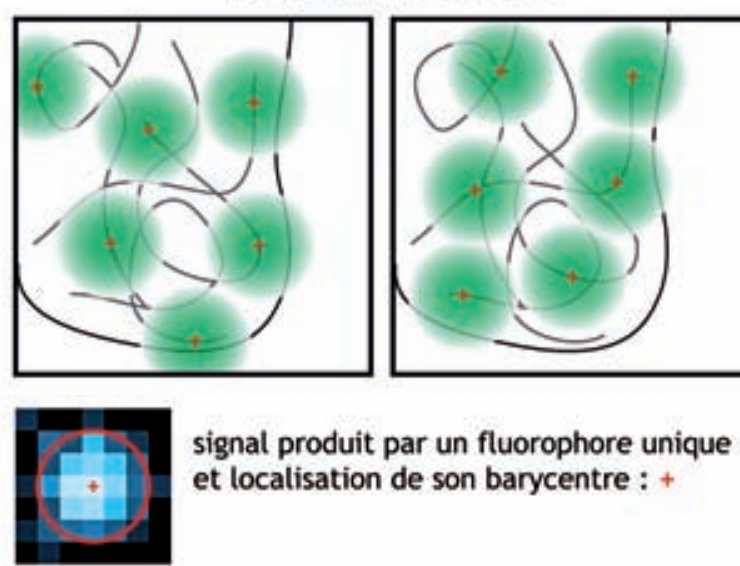

signal produit par un fluorophore unique et localisation de son barycentre : +

image plein-champ

limitée par la diffraction

Figure 6. Principe de la microscopie par super-localisation. génération de l'image super-résolue

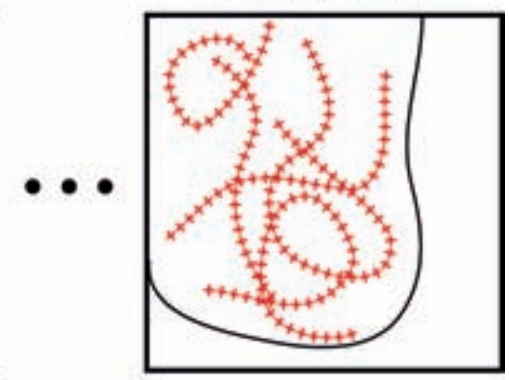

image super-résolue

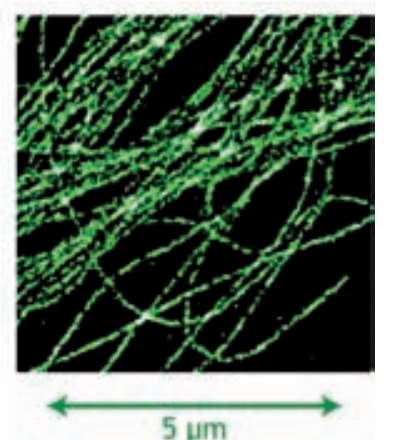

image en fluorescence des sondes activées avec une source appropriée (typiquement $488 \mathrm{~nm}$ pour la version photo-activable de la GFP). Chaque sonde activée produit une tache image de fluorescence dont la taille est donnée par la limite de diffraction. En revanche, le barycentre de cette tache peut être localisé avec une précision bien supérieure à la limite de diffraction: de l'ordre de quelques nanomètres. Une fois que ces sondes activées ont été précisément localisées, elles sont éliminées par photoblanchiment ou renvoyées dans l'état «OFF». Une seconde fraction des sondes est ensuite activée, lue, localisée puis éliminée ou désactivée : ce cycle est répété plusieurs milliers de fois de façon à accéder à la localisation de la quasi-totalité des sondes présentes. L'image PALM est en fait l'image composite obtenue en sommant les contributions des localisations nanométriques relatives à chaque cycle.

Le gain en résolution spatiale est supérieur à un facteur 10 , mais ne concerne que la résolution latérale : la résolution axiale reste a priori inchangée. Afin de diminuer le bruit de fond, c'est-à-dire de s'affranchir du signal de fluorescence provenant des profondeurs de l'échantillon, la microscopie PALM est souvent combinée à un microscope en configuration TIRF. Dans ces conditions, des résolutions spatiales de l'ordre de $20 \mathrm{~nm} \times 20 \mathrm{~nm} \times$ $\sim 100 \mathrm{~nm}(x \times y \times z)$ sont accessibles sur des échantillons fixés, pour un temps d'acquisition total de plusieurs minutes.

Pour ces techniques de super-localisation, le principal challenge concerne la détection d'un signal provenant d'une infime quantité de sondes fluorescentes activées. La faible intensité du signal de fluorescence correspondant contraint fortement la localisation précise du barycentre des taches de diffraction individuelles, donc la résolution spatiale de l'image. Dans le cas d'échantillons vivants, les longues durées d'acquisition de ces images sont peu compatibles avec les mouvements intra-et inter-cellulaires: les meilleures résolutions spatiales obtenues jusqu'ici sont de l'ordre de $70 \mathrm{~nm}$ dans les deux directions transversales.

\section{Conclusion}

Quelques années à peine après leur première mise en œuvre, les techniques de super-résolution ont déjà eu un impact clair sur la biologie cellulaire moderne. Bien que toutes représentent une avancée formidable sur la microscopie conventionnelle, chacune dispose d'avantages et d'inconvénients qui lui sont spécifiques. À I'heure actuelle, il n'existe pas de système idéal qui combine une excellente résolution en trois dimensions et l'imagerie d'échantillons multi-marqués avec des temps d'acquisition et des puissances lumineuses compatibles avec l'étude de cellules vivantes. Dans l'avenir, il sera bien sûr possible d'améliorer les techniques actuelles avec des détecteurs plus sensibles ou des systèmes de commutation plus rapides, mais on conçoit bien que ces futurs développements s'appuieront aussi sur la mise au point de sondes fluorescentes plus efficaces et/ou aux propriétés photo-physiques de commutation toujours plus pointues. Le principal défi restera alors sans doute l'imagerie super-résolue en profondeur des échantillons biologiques: dans ce domaine, les techniques utilisant l'optique adaptative sont prometteuses.

\section{Références}

Stefan Hell, Far-Field Optical Nanoscopy, Science 316, pp 1153-1158 (2007).

Bo Huang, Mark Bates and Xiaowei Zhuang, Super-Resolution Fluorescence Microscopy, Annual Review of Biochemistry 78, pp 993-1016 (2009).

Lothar Schermelleh, Rainer Heintzmann and Heinrich Leonhardt, A Guide to Super-Resolution Fluorescence Microscopy, Journal of Cell Biology 190, pp 165-175 (2010). 